\title{
TLR4 ligation induces expression of APRIL molecule in human neutrophils - a preliminary study
}

\author{
Ewa Jabłońska1, Natalia Wawrusiewicz-Kurylonek², Marzena Garley ${ }^{1}$, Adam Krętowski \\ ${ }^{1}$ Department of Immunology, Medical University of Bialystok, Poland \\ ${ }^{2}$ Department of Endocrinology, Diabetology and Internal Medicine, \\ Medical University of Bialystok, Poland
}

\begin{abstract}
In the present study we investigate the consequences of TLR4 activation by LPS for the synthesis of a proliferation-inducing ligand (APRIL) by human neutrophils (PMNs), and the possible role of the ERK1/2 kinases signaling pathway. In order to make a comparison, the same examinations were carried out on autologous peripheral blood mononuclear cells (PBMCs). The levels of mRNA for APRIL and TLR4 were measured using the real-time PCR method. Western blot analysis was used to assay the expressions of APRIL and ERK1/2 in cell lysates. We discovered an increased expression of APRIL accompanying the increased expression of TLR4 in the LPS-stimulated PMNs and PBMCs. Furthermore, stimulation with LPS triggered similar changes in phospho-ERK1/2 proteins expression in those cells. The present study suggests that LPS plays a role in TLR4-ligation in APRIL induction through ERK1/2 pathway activation in human neutrophils and mononuclear cells of peripheral blood. The association between TLR4 activation and APRIL expression in examined leukocytes might have important implications for the immune response of the host exposed to TLR4 ligands such as LPS. (Folia Histochemica et Cytobiologica 2012, Vol. 50, No. 2, 196-202)
\end{abstract}

Key words: neutrophils, mononuclear cells, proliferation-inducing ligand, toll-like receptor 4, extracellular signal-regulated kinases $1 / 2$

\section{Introduction}

Toll-like receptor 4 (TLR4) is a member of the pattern recognition receptors family (PRRs), recognizing pathogens components, known as pathogen-associated molecular patterns (PAMPs) [1,2]. TLR4 is essential for responses to LPS, a major constituent of the outer membrane of Gram-negative bacteria [1]. It has also been reported that TLR4 is involved in the recognition and elimination of respiratory syncytial virus (RSV) and C. albicans $[1,2]$. Furthermore, TLR4 has been shown to recognize endogenous ligands, including heat shock proteins (HSP60 and

Correspondence address: E. Jabłońska,

Department of Immunology,

Medical University of Bialystok,

Waszyngtona Str. 15A, 15-269 Bialystok, Poland;

tel./fax: + 488574505 46;

e-mail: ewa.jablonska@umwb.edu.pl
HSP70), oligosaccharides of hyaluronic acid, heparin sulfate and fibrinogen [2].

TLR4, similarly to TLR1, TLR2, TLR5, TLR6 and TLR11, is expressed on the immune cell surface, involving neutrophils $[1,3,4]$. Human neutrophils (PMNs), the primary effector cells of the early phase of the immune response, express not only TLR4 but also the majority of identified TLRs, which trigger the different biological functions of those cells [4].

It is well documented that TLR4 activation in neutrophils by LPS up-regulates chemokine receptor expression and function, promotes adhesion, degranulation and phagocytosis, and enhances the production of reactive oxygen species and a wide range of cytokines by these cells [5-7].

Available data indicates that the recognition of specific ligands by different TLRs may be responsible for changes in the ability of different immune cells to secrete cytokines belonging to TNF superfamily 
proteins such as TNF-related apoptosis-inducing ligand (TRAIL), B-cell activating factor (BAFF/Blys), and a proliferation-inducing ligand (APRIL) [8-12]. These TNF superfamily proteins play diverse roles in the proliferation, growth and survival of normal and pathological cells [10, 13-15]. For example, TRAIL exerts a tumor-inhibiting activity associated with selective induction of apoptosis in tumor cells [15]. In contrast, APRIL and BAFF demonstrate a tumorpromoting activity through the inhibition of apoptosis in neoplastic cells [13].

In human neutrophils, the TLR4 receptor has been shown to induce the synthesis of TRAIL [11]. Studies by Scapini et al. indicate that TLR4 receptor does not influence the BAFF gene and protein expression in neutrophils [10]. There is no data available on the regulation of APRIL expression in neutrophils.

In contrast to other molecules belonging to the TNF superfamily, APRIL, also known as TNFSF13 or TALL-2, only exists as a secreted soluble ligand [13]. The APRIL molecule is closely related to BAFF/Blys that binds TACI and BCMA, but not to BAFF-R, which are BAFF-specific receptors [13]. APRIL has been shown to bind heparin sulfate proteoglycans (HSPG) on cell surfaces as well as in the extracellular matrix [16]. Kimberley et al. have suggested that proteoglycan binding domain of APRIL serves as a platform for ligand multimerization and cross-linking [17].

The biological effect of TLR4 ligation is usually associated with the activation of four major signaling pathways: nuclear factor $(\mathrm{NF}-\kappa \mathrm{B})$ pathway and mitogen-activated protein kinases (MAPKs) pathways; the extracellular signal-regulated kinase (ERK); c-Jun $\mathrm{NH}_{2}$-terminal kinase (JNK); and p38 mitogen activated protein kinase $[2,18]$. A signaling pathway involving ERK activation appears to play a crucial role in the induction of biological effects of neutrophils, since it contributes to enhanced translocation of NF- $\kappa \mathrm{B}$ [19]. A number of isoforms of ERK have been described; two of them, ERK-1 (p44MAPK) and ERK-2 (p42MAPK), are expressed in neutrophils and play a role in the regulation of their functions [20].

Taking the above into consideration, in the present study we have investigated the consequences of TLR4 ligation by LPS on the APRIL induction in human neutrophils and, for comparison, in autologous mononuclear cells of peripheral blood (PBMCs). Obtained results suggest that TLR4 activation may lead to an elevated expression of APRIL molecule in human neutrophils and mononuclear cells through ERK-1/2 kinases signaling pathway.

\section{Material and methods}

Isolation of PMNs and PBMCs. The cells were isolated from human whole blood treated with EDTA by way of density centrifugation, using Polymorphprep (Axis-Shield, Oslo, Norway) (density $-1.113 \mathrm{~g} / \mathrm{ml}$ ). This method enables simultaneous separation of two highly purified leukocyte fractions: polymorphonuclear cells, involving neutrophils (PMNs) and mononuclear cells (PBMCs). The purity of isolated PMNs and PBMCs was determined by May-Grunewald-Giemsa-staining.

After washing in PBS without $\mathrm{CaCl}_{2}$ and $\mathrm{MgCl}_{2}$ (Gibco, Paisley, UK) the PMNs were separated by magnetic selection.

CD16 positive neutrophils separation. Neutrophils were separated by positive selection using a Midi MACS magnetic separation system (Miltenyi Biotec, Germany). Isolated cells were suspended in MACS Buffer (up to $5 \times 10^{7}$ total cells) and incubated with CD16 MicroBeads for 30 $\min$ at $4-8^{\circ} \mathrm{C}$. After washing, the cells were suspended in MACS Buffer.

Culture of neutrophils and mononuclear cells. The separated neutrophils and mononuclear cells were suspended in the culture medium (RPMI-1640) to provide $5 \times 10^{6}$ cells $/ \mathrm{ml}$ and incubated in flat-bottomed 96-well plates (Microtest III-Falcon, Franklin Lakes, NJ, USA) for $20 \mathrm{~h}$ at $37^{\circ} \mathrm{C}$ in a humidified incubator with $5 \% \mathrm{CO}_{2}\left(\mathrm{NUAIRE}^{\mathrm{Tw}}\right)$. LPS (10 ng/ml; Sigma) was tested to stimulate the expression of TLR4 and APRIL in both kinds of leukocytes. The viability of neutrophils measured after incubation was $72 \%$, mononuclear cells $-76 \%$, as determined by trypan blue exclusion.

To investigate the role of ERK1/2 kinase in the APRIL synthesis mediated through TLR4 ligation, we assessed the activation thereof by determining the phosphorylation status of this kinase in LPS-stimulated cells by using p42/p44 MAPK inhibitor PD98059 (2'-amino-3'-methoxyflavone), a peptid that specifically inhibits ERK1/2 activation. After isolation, the cells were treated with PD98059 (Calbiochem, Bad Soden, Germany) (40 $\mu \mathrm{M})$ for one hour before LPS stimulation. The presence of inhibitor did not affect cell viability.

RNA isolation and cDNA synthesis. For real-time PCR, total RNA was isolated from $10^{7}$ untreated and stimulated neutrophils or peripheral blood mononuclear cells (RNeasy Mini Kit, Qiagen, Hilden, Germany), according to the manufacturer's specification. The quantity of RNA was verified by spectrophotometry (QuantGen Biopharmacia). RNA integrity was verified by $1.5 \%$ agarose gel electrophoresis identified by ethidium bromide staining and $\mathrm{OD}_{260 / 280} \mathrm{ab}-$ sorbance ratio $>1.95$. One microgram of total RNA was used to prepare cDNA. cDNA synthesis was performed using SuperScript ${ }^{\mathrm{TM}}$ First-Strand Synthesis System for 
RT-PCR (Invitrogen), according to the manufacturer's specification in the MJ Research Thermal Cycler (Model PTC-200, Watertown, MA, USA).

Real-time PCR. Transcript levels were measured by realtime PCR using human genes QuantiTec Hs_TLR4_2_SG Assay (Qiagen), QuantiTec Hs_TNFSF13_2_SG Assay (Qiagen) and QuantiTec Hs_PRS18_1_SG Assay (s18) (Qiagen) as normalizer.

Real-time PCR was performed in duplicate in $20 \mu \mathrm{l}$ using the QuantiTect SYBR Green PCR Master Mix (Qiagen) following the manufacturer's instructions, and carried out in the Chromo4 Real-time PCR Detector (Bio-Rad Laboratories, Hercules, CA, USA). The thermal cycling conditions included an initial activation step at $95^{\circ} \mathrm{C}$ for $15 \mathrm{~min}$ utes, followed by 40 cycles of denaturation, annealing and amplification $\left(95^{\circ} \mathrm{C}\right.$ for $30 \mathrm{~s}, 55^{\circ} \mathrm{C}$ for $30 \mathrm{~s}, 72^{\circ} \mathrm{C}$ for $\left.30 \mathrm{~s}\right)$. At the end of the amplification phase, a melting curve analysis was carried out on the product. Fluorescent data collection was performed during the annealing step.

A standard curve structure was generated employing a series of four dilutions of cDNA, derived from unstimulated cells in reaction with the house-keeping gene - s18. Based on those curves, the levels of total TLR4 and TNFSF13 transcripts were calculated after normalization of TLR4 and TNFSF13 products to $\mathrm{s} 18$. The value of $\mathrm{C}_{\mathrm{T}}$ was determined by the first cycle number at which florescence was greater that the set threshold value. To calculate our data, we used the comparative $\mathrm{C}_{\mathrm{T}}$ method for relative quantification $\left(\Delta \mathrm{C}_{\mathrm{T}}\right.$ method $)$.

Western blot. Cytoplasmic protein fractions of neutrophils and mononuclear cells were analyzed with the use of Western blot for APRIL protein expression and the activation of ERK1/2 kinases by determining their phosphorylation status.

Cells were lysed directly in the presence of Protease Inhibitor Cocktail (Sigma-Aldrich, Steinheim, Germany) by sonication, using Vibra-Cell Ultrasonic Processor (Sonics \& Materials, Inc., USA). Protein fractions were suspended in Laemmli buffer (Bio-Rad Laboratories, Hercules, CA, USA) and next electrophorezed on SDS-PAGE. The resolved protein was transferred onto $0.2 \mu \mathrm{m}$ pore-sized nitrocellulose (Bio-Rad Laboratories, Hercules, CA, USA). The nitrocellulose was incubated at $4^{\circ} \mathrm{C}$ for $4 \mathrm{~h}$ with the respective primary polyclonal antibody: anti-APRIL (R\&D Systems), anti-phospho ERK1/2 (1:100; Santa Cruz Biotechnology, Heidelberg, Germany).

After washing in $0.1 \%$ TBS-T, the membrane was incubated at room temperature for one hour with alkaline phosphatase anti-mouse IgG Abs (Vector Laboratories, Burlingame, CA, USA). Immunoreactive protein bands were visualized following the addition of BCIP/NBT Liquid Substrate System (Sigma-Aldrich, Steinheim, Germany), determined using IMAGEJ (public domain, Java-based image process- ing program developed at the National Institutes of Health, USA) software and estimated by arbitrary units (AU).

The antibody against beta-actin (1:1000; Santa Cruz Biotechnology), which detects the expression of beta-actin in cells lysates, was used as an internal control.

Statistical analysis. Statistical analysis was performed with a statistical package — Statistica 6.0 software. Nonparametric U Mann-Whitney test was used. Results were expressed as median, minimum, and maximum values. For analysis correlation, Pearson's linear correlation was used. P values below 0.05 were considered to be statistically significant.

\section{Results \\ mRNA expression of APRIL and TLR4 analyzed by real-time $P C R$}

Real-time-PCR analysis revealed increased relative expression of APRIL-mRNA levels in neutrophils stimulated with LPS, compared to unstimulated cells. Similarly to APRIL, mRNA levels of TLR4 also increased in these cells (Figure 1).

Treatment of mononuclear cells with LPS also led to elevated APRIL-mRNA and TLR4-mRNA expressions. It is important to note that the expressions of APRIL-mRNA as well as TLR4-mRNA in PBMCs were significantly lower than in PMNs (Figure 1).

Analysis of relationships between APRIL and TLR4 expressions showed correlations in neutrophils and mononuclear cells treated with LPS $(\mathrm{r}=0.79$, $\mathrm{p}<0.05$ and $0.91, \mathrm{p}<0.05$ respectively)

\section{Expressions of APRIL and phospho-ERK1/2 assessed by the Western blot method}

Western blot analysis showed that the lysates of neutrophils and mononuclear cells contained a $27 \mathrm{kDa}$ protein that was stained by anti-APRIL polyclonal antibody (Figure 2A). The obtained results demonstrated significantly enhanced expression of APRIL in examined cells stimulated with LPS compared to unstimulated cells. However, the expression of APRIL in unstimulated and LPS-stimulated neutrophils was lower than in autologous mononuclear cells.

To measure the mechanism of LPS-induced APRIL expression, levels of ERK1/2 kinases (42 and $44 \mathrm{kDa}$ ) were detected. Exposure of neutrophils and mononuclear cells to LPS increased the levels of phosphorylated ERK1/2 (Figure 2B). Similarly to APRIL detected by Western blot analysis, the expression of ERK1/2 in neutrophils was lower than in autologous mononuclear cells.

To confirm the participation of ERK1/2 pathway in the induction of APRIL expression in examined 


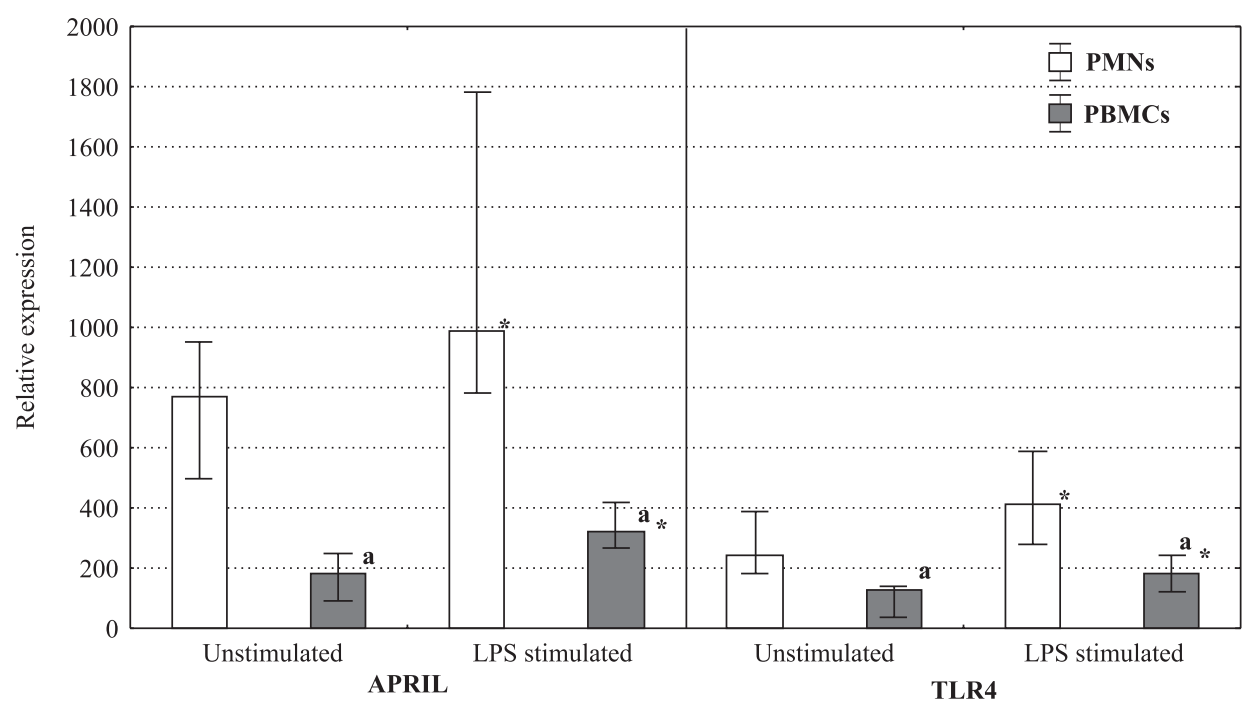

Figure 1. Effects of lipopolysaccharide (LPS) on the expression of APRIL-mRNA and TLR4-mRNA. RT-PCR was performed on human neutrophils and mononuclear cells from peripheral blood. mRNA of TLR4 and APRIL levels were normalized to house-keeping gene - s18. *statistically significant differences between unstimulated and stimulated cells

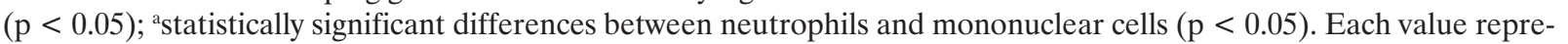
sents the median \pm minimum and maximum for $\mathrm{n}=10$

leukocytes treated with LPS, the levels of phosphorylated ERK1/2 and APRIL expressions in the presence of a selective inhibitor of this pathway (PD98059) were assessed. Increased APRIL and ERK1/2 proteins expression induced by LPS were abrogated in the presence of PD98059 (Figures 2A, B). This observation confirms that ERK1/2 play the role of a critical messenger in TLR4-mediated induction of APRIL.

\section{Discussion}

Available data concerning the regulation of APRIL expression in the immune cells showed that this molecule was detected in monocytes/macrophages and dendritic cells treated with IFNs, CD40L or TLR agonists $\mathrm{CpG}$ and poly(IC) $[12,14]$.

The current study has provided the first evidence that activation of TLR4 receptor is essential for the synthesis of APRIL in human neutrophils. Increased relative expression of APRIL-mRNA accompanied the increased relative expression of TLR4-mRNA in response to LPS treatment. Similar changes were found in autologous mononuclear cells, but expressions of mRNA for APRIL, as well as for TLR4 in those cells, were significantly lower than those in neutrophils. The presence of correlations between mRNA for APRIL and TLR4 in both types of leukocytes appears to confirm the influence of TLR4 ligation by LPS on the expression of APRIL molecule.
Higher expression of TLR4 in neutrophils than in mononuclear cells suggests major sensitivity of neutrophils on a specific ligand - LPS — which can lead to more enhanced effects mediated by this receptor. Similar to TLR4-mRNA, the differences in APRIL expression between examined leukocytes demonstrated in the study appear to confirm this assertion.

Data including changes in APRIL expression at the mRNA levels, analyzed by real time-PCR, differed from the results obtained at the protein level by Western blot analysis. Conversely to mRNA levels, the expression of APRIL protein in PMNs was lower than in PBMCs. A possible explanation of these differences may be the more effective mechanism responsible for processing APRIL in PBMCs than that in PMNs. It is known that APRIL is processed by furin pro-protein convertase inside the Golgi apparatus in myeloid cells, prior to the creation of the form that is then secreted as bioctive soluble molecule [14]. It is probable that the activity of this convertase in PMNs is lower than in PBMCs. Further examinations may be helpful in clarifying this issue. However, taking into consideration the quantitative dominance of neutrophils in the peripheral blood, the expression of APRIL at mRNA and protein levels in those cells may have important implications for the immune response of host exposed to LPS as well as to other exogenous or endogenous ligands for TLR4 receptor [1,2].

Increased expression of APRIL can influence the lifespan and proliferation of B-cells through trans- 


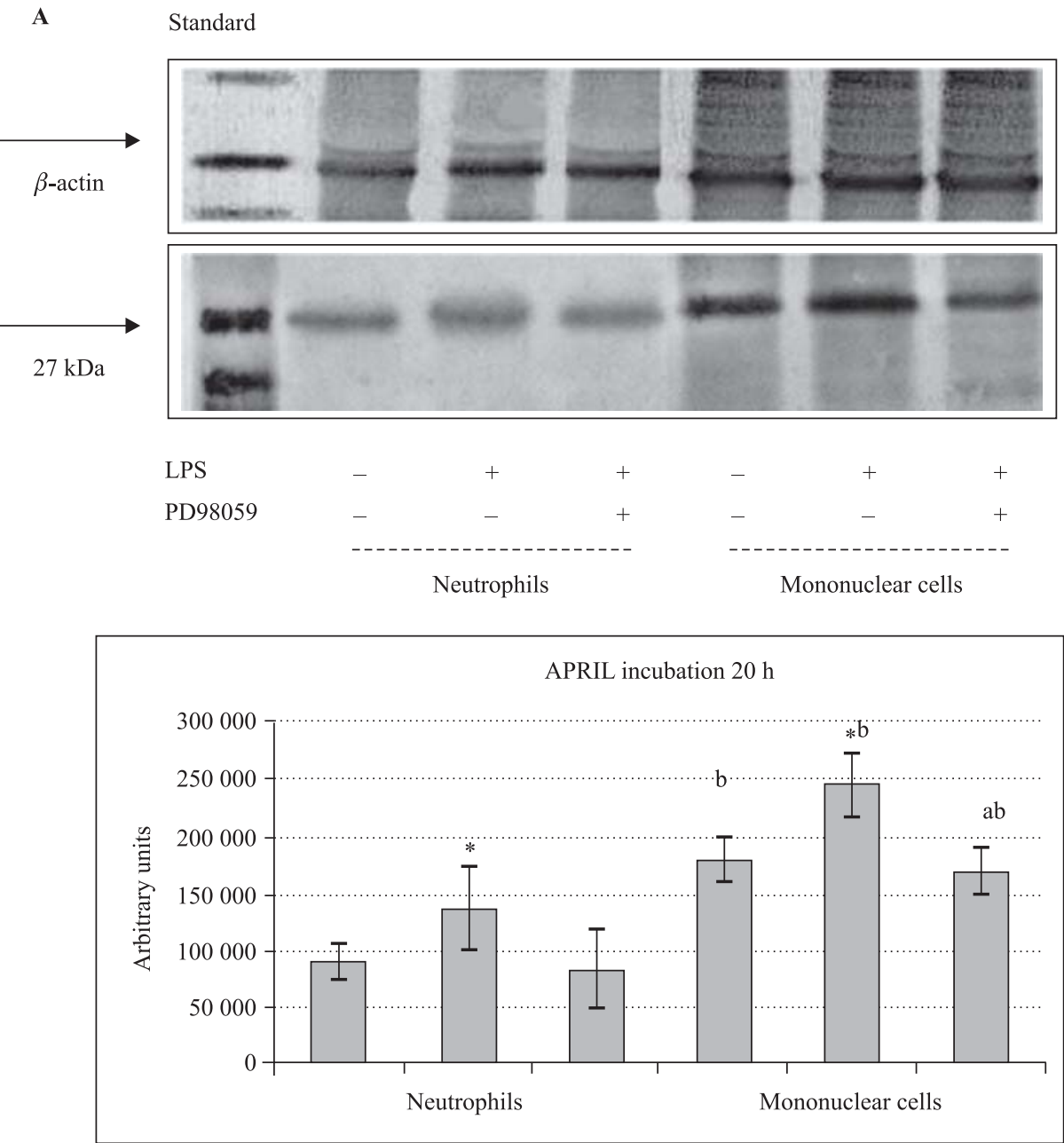

Figure 2A. Western blot analysis of APRIL and phospho-ERK1/2 (B) protein expression in neutrophils and mononuclear cells incubated for $20 \mathrm{~h}$ without or in the presence of LPS and inhibitor PD98059 $(40 \mu \mathrm{M})$. Bands intensity in blot was quantified using Image $\mathbf{J}$ method. *statistically significant differences between unstimulated and stimulated cells

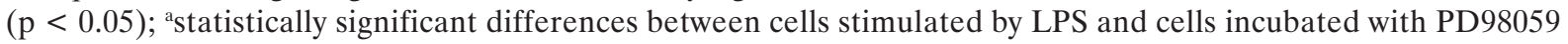
before stimulation with LPS ( $p<0.05$ ); ${ }^{b}$ statistically significant differences between neutrophils and mononuclear cells $(\mathrm{p}<0.05)$

mitting a survival signal into plasmablast/plasma cells. Furthermore, this molecule is involved in the induction of activation-induced deaminase (AID), which is indispensable for Ig-class switching recombinations $[13,21-23]$. These functions suggest that enhanced expression and secretion of APRIL may be involved in autoimmune diseases with a humoral component, such as rheumatic diseases [24]. It has been suggested that neutrophils presenting in the synovium through the APRIL production might play an essential role in the development of rheumatoid arthritis [25].

Elevated synthesis of APRIL by neutrophils and mononuclear cells in response to stimulation by LPS, as presented in the current study, may also impact the cellular immune response. It has been demonstrated that APRIL may up-regulate cofactors such as CD40, MHC II and B7.1 and B7.2 on B-cells, which facilitate antigen presentation to T-cells and lead to their activation, proliferation and survival $[16,21]$.

High expression of APRIL triggered by TLR4 ligation can also affect cancer development. Available data indicates tumor-promoting activity of APRIL, not only in various B-cell malignancies but also in human cancer of colon, thyroid or breast [26-29]. Moreaux et al. demonstrated that APRIL activated NF- $\kappa$ B, PI-3kinase/AKT, and MAPK kinase pathways in myeloma cells and induced a strong up-regulation of the antiapoptotic proteins Mcl-1 and Bcl-2 [28]. Gupta et al. reported that APRIL mediates follicular lymphoma B-cell proliferation [29]. Furthermore, APRIL, through binding heparin sulfate proteoglycans (HSPG) on the surface of tumor cells, may ini- 


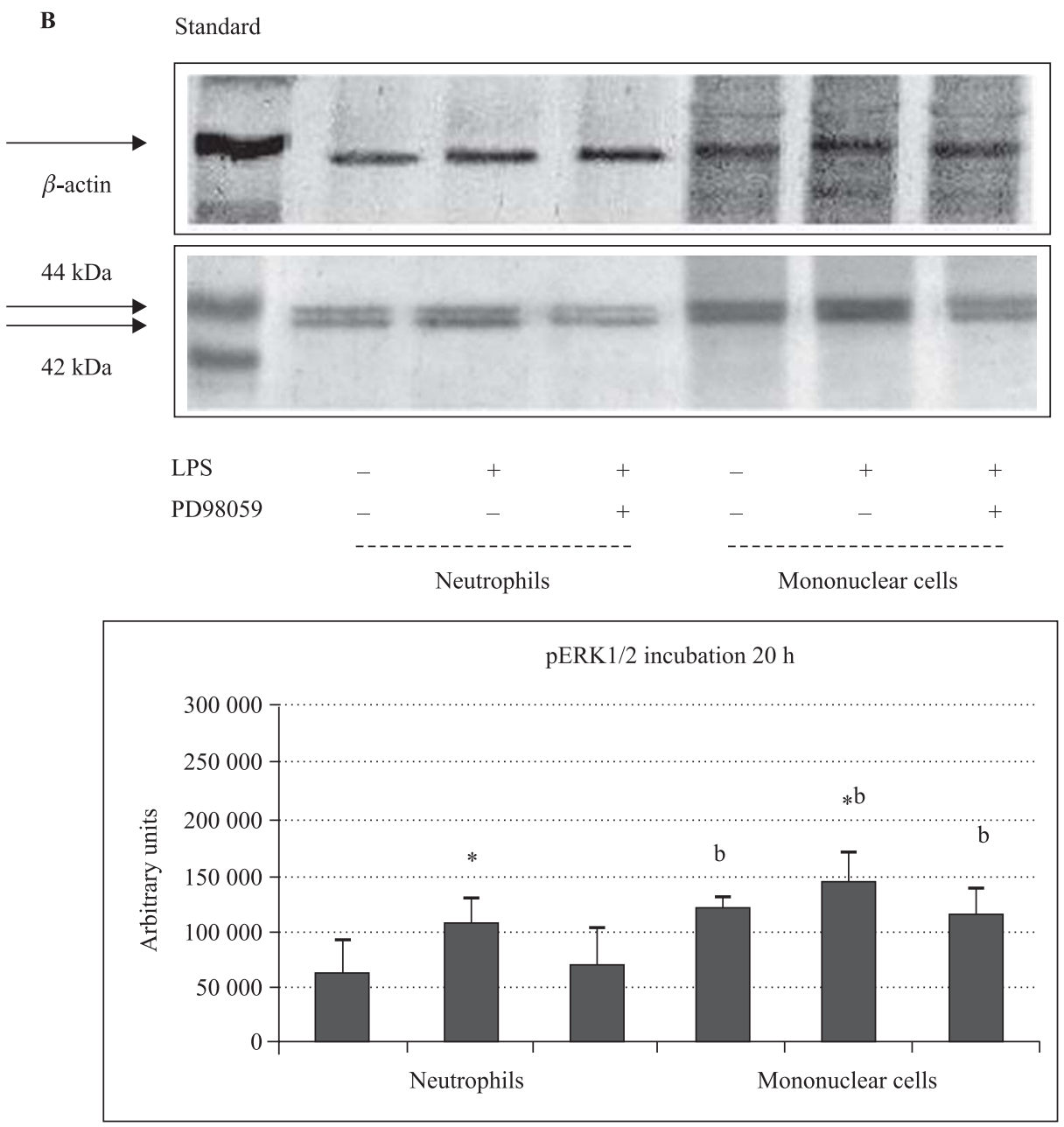

Figure 2B. Western blot analysis of phospho-ERK1/2 protein expression in neutrophils and mononuclear cells incubated for $20 \mathrm{~h}$ without or in the presence of LPS and inhibitor PD98059 $(40 \mu \mathrm{M})$. Bands intensity in blot was quantified using Image $\mathbf{J}$ method. *statistically significant differences between unstimulated and stimulated cells $(\mathrm{p}<0.05)$; ${ }^{\text {astatistically }}$ significant differences between cells stimulated by LPS and cells incubated with PD98059 before stimulation with LPS

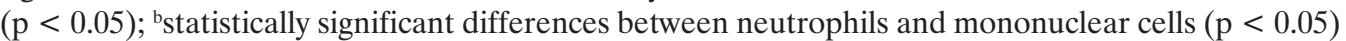

tiate a solid tumor growth through the regulation of tumor cells adhesion and invasion [16, 17].

To explain the mechanism of LPS-induced APRIL expression, we proved that LPS activates the expression of APRIL in human neutrophils and mononuclear cells via ERK1/2 kinases signaling pathway. Similarly to APRIL, the expressions of phospho-ERK1/2 proteins in PMNs were significantly lower than in PBMCs.

The above differences appear to confirm the more effective role of PBMCs than PMNs in forming a biologically active APRIL molecule. Additionally, upregulation of ERK1/2 phosphorylation in the cells treated with LPS may cause enhancement of NF- $\kappa \mathrm{B}$ translocation and transactivation, leading to more transcriptional activation of cytokine genes, involving APRIL in examined leukocytes [30, 31].

\section{Conclusions}

Our preliminary study suggests a potential role of TLR4 ligation by LPS in the ERK1/2-mediated expression of APRIL in human neutrophils, as well as mononuclear cells of peripheral blood. Those observations seem to indicate a new aspect of functions of these leukocytes associated with TLR4 activation. Further research, involving patients with autoimmune or cancer diseases, may confirm the potential significance of the presented findings.

\section{References}

1. Akira S, Uematsu S, Takeuchi O. Pathogen recognition and innate immunity. Cell. 2006;124:783-801.

2. Takeda K, Akira S. Toll-like receptors in innate immunity. Int Immunol. 2005;17:1-14. 
3. Kawai T, Akira S. The roles of TLRs, RLRs and NLRs in pathogen recognition. Int Immunol. 2009;21:317-337.

4. Hayashi F, Means TK, Luster AD. Toll-like receptors stimulate human neutrophil function. Blood. 2003;102:2660 $-2669$.

5. Parker LC, Whyte MKB, Dower SK, Sabroe I. The expression and roles of Toll-like receptors in the biology of the human neutrophil. J Leuk Biol. 2005;77:886-892.

6. Sabroe I, Prince LR, Jones EC et al. Selective roles for Toll-like receptor (TLR) 2 and TLR4 in the regulation of neutrophil activation and life span. J Immunol. 2003;170:5268-5275 .

7. Muzio M, Mantovani A. Toll-like-receptors. Microbes Infect. 2000;2:251-255.

8. Diehl GE, Yue HH, Hsieh K et al. TRAIL-R as a negative regulator of innate immune cell responses. Immunity. 2004;21:877-889.

9. Moon EY, Park H. B cell activating factor (BAFF) gene promoter activity depends upon co-activator, p300. Immunobiology. 2007;212:637-645.

10. Scapini P, Bazzoni F, Cassatella MA. Regulation of B-cell-activating factor (BAFF)/Blymphocyte stimulator (BLyS) expression in human neutrophils. Immunol Let. 2008;116:1-6.

11. Kemp TJ, Ludwig AT, Earel JK et al. Neutrophils stimulation with Mycobacterium bovis bacillus Calmette-Guerin (BCG) results in the release of functional soluble TRAIL/ /Apo-2L. Immunobiology. 2005;106:3474-3482.

12. Hardenberg G, Planelles L, Schwarte CM et al. Specific TLR ligands regulate APRIL secretion by dendritic cells in a PKR-dependent manner. Eur J Immunol. 2007;37:2900-2911.

13. Mackay F, Ambrose C. The TNF family members BAFF and APRIL: the growing complexity. Cytokine Growth Factor Rev. 2003;14:311-324.

14. Dillon SR, Gross JA, Ansel SM, Novak AJ. An APRIL to remember: novel TNF ligands as therapeutic targets. Nat Rev Drug Discov. 2006;5:235-246.

15. MacFarlane M. TRAIL-induced signalling and apoptosis. Toxicol Lett. 2003;139: 89-97.

16. Ingold K, Zumsteg A, Tardivel A et al. Identification of proteoglycans as the APRIL-specific binding partners.J Exp Med. 2005;201:1375-1383.

17. Kimberly FC, van Bostelen L, Cameron $\mathrm{K}$ et al. The proteoglycan (heparin sulfate proteoglycan) binding domain of APRIL serves as a platform for ligand multimerization and cross-linking. FASEB J. 2009;23:1584-1595.

18. Zhou X, Gao XP, Fan J, Liu Q, Anwar KN, Frey RS, Malik AB. LPS activastion of Toll-like receptor 4 signals CD11/CD18 expression in neutrophils. Am J Physiol Lung Cell Mol Physiol. 2005;288:L655-L662.
19. Klein JB, Buridi A, Coxon PY et al. Role of extracellular signal-regulated kinase and phosphatidylinositol-3 kinase in chemoattractant and LPS delay of constitutive neutrophil apoptosis. Cell Signal. 2001;13:335-343.

20. Torres M, Hall FL, O'Neill K. Stimulation of human neutrophils with formyl-methionyl-leucyl-phenylalanine induces tyrosine phosphorylation and activation of two distinct mitogenactivated protein-kinases. J Immunol. 1993;150:1563-1577.

21. Stein JV, López-Fraga M, Elustondo FA et al. APRIL modulates B and T cell immunity. J Clin Invest. 2002;109:1587-1598 .

22. Castigli E, Scott S, Dedeoglu F, Bryce P, Jabara H, Bhan AK. Impaired IgA class switching in APRIL-deficient mice. Proc Natl Acad Sci USA. 2004;101:3903-3908.

23. Bossen C, Cachero TG, Tardivel A et al. TACI, unlike BAFF-R, is solely activated by oligomeric BAFF and APRIL to support survival of activated B cells and plasmablasts. Blood. 2008;111:1004-1012.

24. Mackay F, Sierro F, Grey ST, Gordon TP. The BAFF/APRIL system: an important player in systemic rheumatic diseases. Curr Dir Autoimmun. 2005;8:243-265.

25. Gabay C, Krenn V, Bosshard C, Seemayer CA, Chizzolini C, Huard B. Synovial tissues concentrate secreted APRIL. Arthritis Res Ther. 2009;11:R144.

26. Hendriks J, Planelles L, de Jong-Odding J et al. Heparan sulfate proteoglycan binding promotes APRIL-induced tumor proliferation. Cell Death Differ. 2005;12:637-648.

27. Pelekanou V, Kampa M, Kafousi M et al. Expression of TNF-superfamily members BAFF and APRIL in breast cancer: immunohistochemical study in 52 invasive ductal breast carcinoma. BMC Cancer. 2008;8:76.

28. Moreaux J, Vevrune JL, De Vos J, Klein B. APRIL is overexpressed in cancer link with tumor progression. BMC Cancer. 2009;9:83.

29. Gupta M, Dillon SR, Ziesmer SC et al. A proliferation-inducing ligand mediates follicular lymphoma B-cell proliferation and D1 expression through phosphatidylinositol 3-kinaseregulated mammalian target of rapamycin activation. Blood. 2009;113:5206-5216.

30. Cloutier A, Ear Th, Blais-Charron E, Dubois CM, McDonald PM. Differential involvement of NF-kB and MAP kinase pathways in the generation of inflammatory cytokines by human neutrophils. J Leukoc Biol. 2007; 81:567-577.

31. Wu TT, Chen TL, Loon WS, Tai YT, Cherng UG, Chen RM. Lipopolysaccharide stimulates synthesis of toll-like receptor 2 and surfactant protein-A in human alveolar epithelial A549 cells through upregulating phosphorylation of MEK1 and ERK1/2 and sequential activation of NF-kB. Cytokine. 2011; $55: 40-47$. 